\title{
Letter regarding Wang et al. entitled "Effects of murine double minute 2 polymorphisms on the risk and survival of osteosarcoma: a systemic review and meta-analysis"
}

\author{
Guoyan Liu • Wanli Xu • Yanke Hao • Zhanwang Xu
}

Received: 18 November 2013 / Accepted: 2 December 2013 / Published online: 21 December 2013

(C) International Society of Oncology and BioMarkers (ISOBM) 2013

To the Editor,

Wang et al. [1] conducted a meta-analysis to assess the effects of murine double minute 2 (MDM2) polymorphisms on osteosarcoma risk and survival of patients with osteosarcoma. They calculated the pooled odds ratio (OR) or hazard ratio (HR) with $95 \%$ confidence intervals (95\% CIs) by using different effects models and concluded that MDM2 polymorphisms have some effects on the risk of osteosarcoma but have no effect on the survival of patients with osteosarcoma. Before their results can be accepted, we would like to express some concerns in relation to their meta-analysis.

Firstly, the investigators clarified that "PubMed, Web of Science, and Wanfang databases were searched for eligible studies" in the "Abstract" section. However, they searched the PubMed and EMBASE databases for eligible studies in the "Methods" section. We would like to know the possible reason for this difference. To make the article more credible, the investigators should give us the real search issues. The small number of required papers would be an important limitation of the review. We suggest that more electronic databases be systematically searched.

Secondly, the investigators included only one study on the association between MDM2 rs2279744 polymorphism and the overall survival of patients with osteosarcoma, which could not reflect the real association between them. In our

G. Liu $\cdot$ Y. Hao $\cdot$ Z. Xu $(\bowtie)$

Department of Orthopaedics, Affiliated Hospital, Shandong

University of Traditional Chinese Medicine,

Jinan 250014, Shandong Province, China

e-mail: zhanwang1212@126.com

W. Xu

College of Basic Medical Sciences, Shandong University of

Traditional Chinese Medicine, Jinan 250355,

Shandong Province, China opinion, the investigators should search more electronic databases to find more studies.

Thirdly, with respect to the data extraction, two investigators independently extracted data and reached a consensus. We would like to know how problems were solved if there were discrepancies between the two investigators.

Fourthly, we suggest that the investigators evaluate the methodological quality of the selected studies, which could avoid potential bias in the meta-analysis. Each included paper could be independently assessed by two investigators using a standardized electronic form of predefined criteria. Also, the investigators could describe how to evaluate the quality of all studies in the meta-analysis.

Finally, the departure of frequencies of MDM2 polymorphisms from expectation under Hardy-Weinberg equilibrium in the control population should be assessed using the goodness-of-fit chi-square test with a $P<0.05$ considered as significant disequilibrium. A significant difference between the observed and expected genotype frequencies under Hardy-Weinberg equilibrium (HWE) may indicate genotyping error [2]. Departure from HWE can be caused by factors such as inbreeding caused by consanguinity, assortative mating, i.e., nonrandom mating, selection, or migration [3]. Although exceptions to the conditions of HWE may explain deviation, it is critical that investigators recognize the need to perform a test of HWE and then evaluate the reasons for any observed deviation.

Thanks go to the authors for their contribution of supplying us with an assessment of the effects of MDM2 polymorphisms on osteosarcoma risk and survival of patients with osteosarcoma. However, further studies with large samples are still needed to assess the effects of MDM2 polymorphisms on the risk and survival of osteosarcoma.

Conflicts of interest None 


\section{References}

1. Wang LC, Liu ZH, Jing PW, Shao L, Chen L, He X, et al. Effects of murine double minute 2 polymorphisms on the risk and survival of osteosarcoma: a systemic review and meta-analysis. Tumour Biol. 2013. doi:10.1007/s13277-013-1227-8.
2. Xu J, Turner A, Little J, Bleecker ER, Meyers DA. Positive results in association studies are associated with departure from HardyWeinberg equilibrium: hint for genotyping error? Hum Genet. 2002;111(6):573-4.

3. Ziegler A, Van Steen K, Wellek S. Investigating Hardy-Weinberg equilibrium in case-control or cohort studies or meta-analysis. Breast Cancer Res Treat. 2011;128(1):197-201. 